\section{PWE-015 CLINICAL EFFICACY AND SAFETY OF ANTI-TNF THERAPY IN INFLAMMATORY BOWEL DISEASE IN THE ELDERLY}

Jonathan Digby-Bell*, Hajir Ibraheim, Fakhirah Badrulhisham, Nick Powell. Guy's and St Thomas' Nhs Trust, London, UK

\subsection{6/gutjnl-2018-BSGAbstracts. 147}

Introduction Many patients, especially the elderly, are excluded from clinical drug trials and little real-life data exists on the safety and efficacy of anti-TNF in this comorbid and difficult to treat group. We wanted to compare the clinical efficacy and safety of anti-TNF therapy in patients over 60 years in a tertiary IBD centre in London.

Methods We interrogated our IBD biologics database from January 2009 to November 2015 and performed retrospective data analysis until end of follow up in April 2017. Data was collected on demographics, biochemistry and clinical scores. For every ' $\geq 60$ ' patient identified we randomly selected two '<60' comparators.

Primary endpoints week 14 and week 54 steroid free clinical remission (Harvey Bradshaw Index $<5$ or Simple Colitis Activity index $<3$ ).

Secondary endpoint proportion of patients remaining on antiTNF at the end of follow up

Results 29 patients (23 Crohn's, 4 ulcerative colitis, 1 IBD unclassified) started anti-TNF aged ' $\geq 60$ ' and 58 randomly chosen $<60$ years were selected for analysis.

\begin{tabular}{lll} 
Abstract PWE-015 Table 1 & & \\
\hline & $<60$ years & $\geq 60$ years \\
\hline Week 14 steroid free remission & $28 / 41(68.3 \%)$ & $\mathrm{n}=58$ \\
Week 54 steroid free remission & $24 / 40(60 \%)$ & $8 / 15(53.3 \%)$ \\
Remain on anti-TNF at end of & $38 / 58(65.5 \%)$ & $12 / 29(41.4 \%)$ p $<0.05$ \\
follow up (April 2017) & & \\
Reasons for stopping biologic & 8 primary non- & 4 primary non-response \\
during study period & response & 5 secondary loss of response \\
& 7 secondary loss of & 1 infusion reaction \\
& response & 1 remission \\
& 1 infusion reaction & 1 infection \\
& 1 remission & 1 colorectal cancer \\
& 2 infections & 1 severe fatigue \\
& 1 stopped & 1 peripheral neuropathy \\
& attending & 2 stopped attending \\
Anti-drug antibodies during follow & $3 / 58(5.2 \%)-3$ & $4 / 29(13.8 \%)-3$ infliximab, \\
up & infliximab & 1 adalimumab \\
Adverse events during follow up & 1 new cancer & 3 new cancers \\
& 1 infusion reaction & 1 ileal perforation \\
& 2 infections & 1 infusion reaction \\
& & 2 infections \\
\hline
\end{tabular}

Conclusions Only a small number of ' $\geq 60$ ' patients started anti-TNF (29 out of greater than 650 ). This may reflect our local population, less severe disease or that clinicians favour non anti-TNF therapies in this older group.

Overall there was similar clinical efficacy at weeks 14 and 54 of anti-TNF therapy between the 'young' and 'old' groups.

There was a statistically significantly higher discontinuation rate after 1 year of therapy in the older group $(p=0.043)$.
There were more adverse events in the older group (7/29), including 3 new cancer diagnoses, compared with the younger group $(4 / 58)$.

4 patients had detectable anti-drug antibodies in the older group despite 2 of them having therapeutic thiopurine suggesting that the elderly may have more immunogenicity than the young.

Further studies with more patients across multiple sites are required to clarify safety and efficacy of anti-TNF therapy in the elderly.

\section{PWE-016 DERMATOLOGICAL REACTIONS ASSOCIATED WITH ANTI -TNF THERAPY IN INFLAMMATORY BOWEL DISEASE: AN12 YEARS EXPERIENCE}

Rizwan Diyar*, Anirudh Bhandare, Jo Taylor, Jimmy K Limdi. Pennine Acute Hospitals Trust, Manchester, UK

\subsection{6/gutjnl-2018-BSGAbstracts. 148}

Introduction Anti-TNF therapies have significantly influenced our treatment paradigms and are amongst our most effective therapies to induce and maintain remission. They are, however, not without side effects. Data on dermatological complications are limited. Our aim was to describe the incidence, management and outcome of dermatological complications of anti-TNF therapy in IBD patients at our centre.

Methods We conducted a retrospective review of 435 consecutive IBD patients treated with anti-TNF (Infliximab or Adalimumab) therapy between 2005-2017. Data was collected from electronic case records and included demographics, disease characteristics, medical history, treatment and dermatological complications.

Results Forty-four patients (10.1\%) had dermatological complications. Of these, 25 were female (56.8\%). Thirty four patients had Crohn's disease $(C D=77.2 \%)$ and 10 had ulcerative colitis $(\mathrm{UC}=22.8 \%)$. Disease phenotype in $\mathrm{CD}$ was $\mathrm{A} 2$ (24) L3 (22) B1 (21) in respective categories whereas $7 / 10$ UC patients had Montreal E3 disease. Mean age at diagnosis was 34.31 years (median 28,IQR 23.5) Thirty-four patients were treated with Infliximab. The most common reaction reported was a non-specific rash in 11 patients, eczema in 8 and psoriasiform reactions in 7 patients. Shingles was noted in 5 cases and 3 patients had urticaria. Seborrheic dermatitis, cellulitis, itching, acne, and skin abscesses were noted in two patients each along with one case each of Lichen planus and chicken pox.

The average time of onset of symptoms from treatment was 20 months (median 7,IQR 27).

Twenty of 34 patients treated with infliximab, continued treatment following the dermatological side effect without any further reactions, 12 were switched to adalimumab of which 1 patient had a further psoriasiform reaction leading to cessation of Anti TNF therapy and initiation of ustekinumab. One patient was not given any further Anti-TNF treatment after the initial reaction and one patient was switched to ustekinumab after the reaction.

Of 10 patients on adalimumab who had dermatological side effects, all continued on therapy without any further reactions.

Conclusions The incidence of dermatological effects was $10.1 \%$ in our cohort. We did not observe a 'class effect' as 